\title{
Higher frequency of comorbidities in fully vaccinated patients admitted to the ICU due to severe COVID-19: a prospective, multicentre, observational study
}

\begin{abstract}
Copyright $\odot$ The authors 2022 .
This version is distributed under the terms of the Creative Commons Attribution NonCommercial Licence 4.0. For commercial reproduction rights and permissions contact permissions@ersnet.org

Received: 18 Aug 2021 Accepted: 13 Nov 2021

To the Editor:

The coronavirus disease 2019 (COVID-19) vaccination campaign in Spain began on 27 December 2020 [1]. To date, more than 36 million people have been fully vaccinated, with most of the population, namely 25.3 million people (69.1\%), receiving BNT 162b2 (Pfizer/BioNTech) [1]. With respect to other vaccines and figures, 4.8 million (13.2\%) people have received AZD1222 (Oxford/AstraZeneca); 4.5 million (12.3\%) mRNA-1273 (Moderna); and 2.0 million (5.4\%) JNJ-78436735 (Janssen) [1].

Vaccination uptake has radically changed how the severe acute respiratory syndrome coronavirus 2 (SARS-CoV-2) infection has impacted healthcare systems [2, 3]. Since the initiation of the campaign, a total of 19705 patients with severe COVID-19 have required admission to intensive care unit (ICU) in Spain, the vast majority with no vaccination or an incomplete regimen [1]. Although vaccination has been shown to be notably effective, a few fully vaccinated patients can develop severe COVID-19 requiring ICU admission. To our knowledge, there is no description of this cohort of patients.

Within the CIBERESUCICOVID consortium [4], we reported a prospective, multicentre and observational study that characterised fully vaccinated patients admitted to seven Spanish ICUs for severe COVID-19 between 25 January and 14 September 2021. These patients developed COVID-19 symptoms at least 2 weeks after administration of either a single-dose COVID-19 vaccine (JNJ-78436735) or the second dose of a two-dose vaccine. Exclusion criteria for this study included unconfirmed SARS-CoV-2 infection; ICU admission due to other causes; or incomplete vaccination status. Data was collected as previously described [4]. For the purpose of comparison, we included 105 consecutive, non-vaccinated adult patients with laboratory-confirmed SARS-CoV-2 infection requiring admission to the same seven ICUs between 25 January and 13 May 2021.

Continuous variables are reported as median (interquartile range) and compared between groups using the Mann-Whitney test. Categorical variables are reported as frequencies (percentages) and compared using Fisher's exact test.

The study received approval by the institution’s internal review board (Comité Ètic d’Investigació Clínica, registry number HCB/2020/0370), and we obtained informed consent from either patients or their relatives.

During the study period, a total of 1585 patients were admitted to ICUs across seven Spanish hospitals due to COVID-19. Of those, 1314 (82.9\%) were unvaccinated; 161 (10.2\%) had not completed the vaccination regimen; and 110 (6.9\%) were fully vaccinated. Data from 81 (73.6\%) fully vaccinated patients were available for the analysis.

Demographics and clinical characteristics of the fully vaccinated population are detailed in table 1 . In summary, the median age was $68.0(60.0-74.0)$ years; 35 (43.2\%) patients were aged $\geqslant 70$ years, whilst only five patients were $<50$ years. $72 \%(n=58)$ of these patients were male. All of the patients but two had at least one comorbidity, whereas $69.1 \%(n=56)$ had three or more. The most frequent comorbidity was

Shareable abstract (@ERSpublications)

Severe COVID-19 disease requiring ICU admission is possible in the fully vaccinated population, especially in those with immunocompromised status and other comorbidities. Interventions to improve vaccine response might be necessary in this population. https://bit.ly/3Fw6vCP

Cite this article as: Motos A, López-Gavín A, Riera J, et al. Higher frequency of comorbidities in fully vaccinated patients admitted to the ICU due to severe COVID-19: a prospective, multicentre, observational study. Eur Respir J 2022; 59: 2102275 [DOI: 10.1183/13993003.02275-2021].
\end{abstract}


TABLE 1 Characteristics of fully and non-vaccinated, intensive care unit (ICU)-admitted patients with COVID-19

Fully vaccinated patients $(n=81)$

Non-vaccinated patients $(n=105)$

p-value

\begin{tabular}{|c|c|c|c|}
\hline \multicolumn{4}{|l|}{ Baseline characteristics } \\
\hline Age, years & $68.0(60.0-74.0)$ & $65.0(55.0-73.0)$ & 0.24 \\
\hline Male & $58(71.6 \%)$ & $71(67.6 \%)$ & 0.63 \\
\hline BMI, $\mathrm{kg} \cdot \mathrm{m}^{-2}$ & $27.6(24.9-31.7)$ & $30.1(26.5-33.7)$ & 0.010 \\
\hline \multicolumn{4}{|l|}{ Comorbidities } \\
\hline Number of comorbidities & $3(2-4)$ & $2(1-4)$ & 0.005 \\
\hline Hypertension & $61(75.3 \%)$ & $52(49.5 \%)$ & $<0.001$ \\
\hline Chronic cardiac disease & $15(18.5 \%)$ & $15(14.3 \%)$ & 0.55 \\
\hline Chronic respiratory disease $^{\#}$ & $21(25.9 \%)$ & $16(15.2 \%)$ & 0.095 \\
\hline Chronic renal disease & $16(19.8 \%)$ & $10(9.5 \%)$ & 0.055 \\
\hline Obesity $\left(\mathrm{BMI} \geqslant 30 \mathrm{~kg} \cdot \mathrm{m}^{-2}\right)$ & $30(37.0 \%)$ & $57(54.3 \%)$ & 0.026 \\
\hline Diabetes mellitus & $35(43.2 \%)$ & $26(24.8 \%)$ & 0.011 \\
\hline Immunosuppression & $28(34.6 \%)$ & $11(10.5 \%)$ & $<0.001$ \\
\hline Solid organ transplant & $13(46.4 \%)$ & $8(72.7 \%)$ & \\
\hline Active malignancy & $11(39.3 \%)$ & 0 & \\
\hline Autoimmune disease & $3(10.7 \%)$ & $2(18.2 \%)$ & \\
\hline Chronic immunosuppressor treatment & $1(3.6 \%)$ & $1(5.6 \%)$ & \\
\hline Active or former smoker & $30(37.0 \%)$ & $42(40.0 \%)$ & 0.76 \\
\hline \multicolumn{4}{|l|}{ Disease chronology } \\
\hline Days from last vaccine dose to COVID-19 symptoms & $75.0(47.0-95.0)$ & & \\
\hline Days from COVID-19 onset to hospital admission & $6.0(4.0-8.0)$ & $8.0(6.0-10.0)$ & $<0.001$ \\
\hline Days from hospital admission to ICU admission & $1.0(0-3.0)$ & $1.0(0-3.0)$ & 0.20 \\
\hline Days from ICU admission to IMV & $1.0(0-3.0)$ & $0(0-1.0)$ & 0.001 \\
\hline \multicolumn{4}{|l|}{ ICU admission } \\
\hline APACHE II score & $12(9-17)$ & $10(8-13)$ & 0.003 \\
\hline SOFA score & $4(3-5)$ & $4(3-6)$ & 0.64 \\
\hline \multicolumn{4}{|l|}{ Adjuvant treatments } \\
\hline COVID-19 therapies & $28(34.6 \%)$ & $12(11.4 \%)$ & $<0.001$ \\
\hline Remdesivir & $21(75.0 \%)$ & $7(58.3 \%)$ & \\
\hline Tocilizumab & $14(50.0 \%)$ & $3(25.0 \%)$ & \\
\hline Convalescent plasma & $3(10.7 \%)$ & $2(16.7 \%)$ & \\
\hline Subcutaneous heparin & $77(95.1 \%)$ & $104(99.0 \%)$ & 0.17 \\
\hline Low dose ( $\leqslant 1 \mathrm{mg} \cdot \mathrm{kg}^{-1}$ per day) & $61(75.3 \%)$ & $76(73.1 \%)$ & \\
\hline High dose (>1 $\mathrm{mg} \cdot \mathrm{kg}^{-1}$ per day) & $16(19.8 \%)$ & $28(26.9 \%)$ & \\
\hline Vasopressor treatment & $37(45.7 \%)$ & $58(55.2 \%)$ & 0.24 \\
\hline Continuous neuromuscular blockers & $39(48.1 \%)$ & $70(66.7 \%)$ & 0.016 \\
\hline Corticosteroids & $76(93.8 \%)$ & $104(99.0 \%)$ & 0.087 \\
\hline \multicolumn{4}{|l|}{ Supportive therapies } \\
\hline High-flow oxygen cannula & $65(80.2 \%)$ & $56(53.3 \%)$ & $<0.001$ \\
\hline NIMV & $21(25.9 \%)$ & $25(23.8 \%)$ & 0.86 \\
\hline IMV & $45(55.6 \%)$ & $76(72.4 \%)$ & 0.020 \\
\hline Prone position & $42(51.9 \%)$ & $62(59.0 \%)$ & 0.23 \\
\hline ECMO support & $1(1.2 \%)$ & $1(1.0 \%)$ & 1.00 \\
\hline Renal replacement therapy & $10(12.3 \%)$ & $4(3.8 \%)$ & 0.047 \\
\hline Limitation of life-sustaining care & $16(19.7 \%)$ & $7(6.7 \%)$ & 0.012 \\
\hline \multicolumn{4}{|l|}{ Complications } \\
\hline Nosocomial bacterial pneumonia $^{+}$ & $22(27.2 \%)$ & $45(42.9 \%)$ & 0.032 \\
\hline Ventilator-associated pneumonia & $16(72.7 \%)$ & $35(77.8 \%)$ & 0.76 \\
\hline Microbiological diagnosis ${ }^{\S}$ & $18(81.8 \%)$ & $42(93.3 \%)$ & 0.21 \\
\hline Pseudomonas aeruginosa & $7(38.9 \%)$ & $10(23.8 \%)$ & \\
\hline Klebsiella spp. & $4(22.2 \%)$ & $2(4.8 \%)$ & \\
\hline Staphylococcus aureus & $3(16.7 \%)$ & $11(26.2 \%)$ & \\
\hline Acinetobacter baumannii & $2(11.1 \%)$ & $2(4.8 \%)$ & \\
\hline Other & $5(27.8 \%)$ & $20(47.6 \%)$ & \\
\hline Acute renal injury ${ }^{f}$ & $23(28.4 \%)$ & $25(23.8 \%)$ & 0.50 \\
\hline Pulmonary embolism & $6(7.4 \%)$ & $8(7.6 \%)$ & 1.00 \\
\hline Myocardial infarction & $1(1.2 \%)$ & $1(1.0 \%)$ & 1.00 \\
\hline Heart failure & $3(3.7 \%)$ & $2(1.9 \%)$ & 0.65 \\
\hline Stroke & $0(0 \%)$ & $2(1.9 \%)$ & 0.51 \\
\hline Liver dysfunction ${ }^{\# \#}$ & $32(39.5 \%)$ & $32(30.5 \%)$ & 0.22 \\
\hline
\end{tabular}


TABLE 1 Continued

Fully vaccinated patients $(n=81)$

Non-vaccinated patients $(n=105)$

\begin{tabular}{|c|c|c|c|}
\hline \multicolumn{4}{|l|}{ Outcomes } \\
\hline 28-day mortality & $24(29.6 \%)$ & $27(25.7 \%)$ & 0.62 \\
\hline ICU mortality & $27(33.3 \%)$ & $30(28.6 \%)$ & 0.52 \\
\hline In-hospital mortality & $28(34.6 \%)$ & $30(28.6 \%)$ & 0.43 \\
\hline Length of IMV, days & $19.0(9.0-28.0)$ & $20.0(10.0-29.0)$ & 0.51 \\
\hline Length of ICU stay, days & $11.0(7.0-30.0)$ & $15.0(9.0-30.0)$ & 0.044 \\
\hline Length of hospital stay, days & $19.0(14.0-36.0)$ & $21.0(14.0-36.0)$ & 0.31 \\
\hline
\end{tabular}

Continuous variables are reported as median (interquartile range) and categorical variables as frequencies (\%). Sample sizes were indicated for each variable and percentages were calculated in accordance with available data. Missing data were only present for APACHE II and SOFA scores. Specifically, data were available for 171 and 169 patients, respectively. p-values $<0.05$ were considered significant and are shown in bold. APACHE II: Acute Physiology and Chronic Health Evaluation II; BMI: body mass index; ECMO: extracorporeal membrane oxygenation; IMV: invasive mechanical ventilation; NIMV: non-invasive mechanical ventilation; SOFA: Sepsis-related Organ Failure Assessment. \#: chronic respiratory disease includes any of COPD, cystic fibrosis, bronchiectasis, interstitial lung disease, asthma, or pre-existing requirement for long-term oxygen therapy. ฯ. immunosuppression includes current solid organ or haematological malignancy, AIDS/HIV, solid organ transplant, haematopoietic cell transplant, autoimmune diseases and any immunosuppressant treatment taken within 14 days of hospital admission. ${ }^{+}$: clinically or radiologically diagnosed bacterial pneumonia managed with antimicrobials; bacteriological confirmation was not required. \$: three patients had polymicrobial pneumonia in the fully vaccinated group, two in the non-vaccinated group. ${ }^{f}$ : acute renal injury was defined as an increase in serum creatinine by $\geqslant 0.3 \mathrm{mg} \cdot \mathrm{dL}^{-1}$ within $48 \mathrm{~h}$ or as an increase in serum creatinine $\geqslant 1.5$ times more than baseline. \#\#: liver dysfunction was defined as an increase in blood bilirubin, alanine transaminase or aspartate transaminase twice the upper limit of the normal range.

hypertension, being present in 61 (75.3\%) patients. 28 (34.6\%) patients had an immunocompromised status. The percentage of obese (BMI $\geqslant 30 \mathrm{~kg} \cdot \mathrm{m}^{-2}$ ) patients was $37.0 \%(\mathrm{n}=30)$. Patients required ICU admission after a median time of 82.0 (55.0-101.0) days since vaccination, and APACHE II (Acute Physiology and Chronic Health Evaluation II) and SOFA (Sepsis-related Organ Failure Assessment) scores at this time point were $12(9-17)$ and $4(3-5)$, respectively. All patients showed bilateral pulmonary infiltrates. Additionally, 35 of 81 (43.2\%) vaccines administered were BNT 162b2; 26 (32.1\%) JNJ-78436735; 16 (19.8\%) mRNA-1273; and four (4.9\%) AZD1222.

Amongst the fully vaccinated population, 45 (55.6\%) received invasive mechanical ventilation. 42 (51.9\%) patients were placed in the prone position, and only one patient received extracorporeal membrane oxygenation support. All but five (93.8\%) patients received corticosteroids. Furthermore, all patients but four $(95.1 \%)$ received subcutaneous anticoagulation; 72 (88.9\%) underwent antimicrobial therapies. 22 (27.2\%) patients were diagnosed with nosocomial bacterial pneumonia, whilst 23 (28.4\%) patients suffered acute kidney failure.

The in-hospital mortality rate was $34.6 \%$, and the main causes of death included respiratory failure $(\mathrm{n}=19$, $67.9 \%)$ and multiorgan failure $(n=4,14.3 \%)$. The median duration of invasive mechanical ventilation was 19.0 (9.0-28.0) days, and the median length of ICU stay was 11.0 (7.0-30.0) days.

To our knowledge, this study is the first descriptive report of fully vaccinated patients requiring ICU admission due to severe COVID-19. The main finding of this study is that patients with specific comorbidities and full vaccination regimen may be at risk of developing severe COVID-19, even though vaccines have proven to be greatly effective in the general population [2, 3, 5]. Importantly, only $7 \%$ of patients with severe COVID-19 were fully vaccinated. We observed a notably high incidence of comorbidities in this population, especially as they relate with vascular disease (i.e. hypertension, diabetes mellitus and chronic renal disease) and immunosuppression status. When we compared this incidence with that of a non-vaccinated group of patients requiring ICU admission during coinciding periods, we observed a three-fold increase in immunosuppression; chronic respiratory disease, renal disease, diabetes mellitus and hypertension rates almost doubled. Of note, the median time between the onset of symptoms and hospital admission was significantly shorter for fully vaccinated cases than unvaccinated patients with COVID-19.

Contou et al. [6] have described a second-wave French cohort of non-vaccinated patients. This cohort had similar or slightly increased comorbidity rates compared to those of our non-vaccinated group, albeit lower than that of our fully vaccinated patients. Juthani et al. [7] and Brosh-Nissimov et al. [8] have reported small series of fully vaccinated patients that required hospitalisation, including mild to severe patients. Like our study, both investigations found a high rate of comorbidities amongst severe or critically ill patients [7, 8]. 
In a case-control study including 35 fully vaccinated patients admitted to the ICU, TENFORDE et al. [9] found that the significant association between hospitalisation for COVID-19 and decreased likelihood of vaccination was weaker in immunocompromised patients than immunocompetent patients.

The implications of our findings are manifold. First, these findings encourage discussion on the possible need for further interventions, such as the use of COVID-19 vaccine boosters, in this population. Some recent studies have already debated the practicality of a third dose of the vaccine [10-12]. Our data suggest that patients with comorbidities may benefit from these strategies.

Secondly, the substantial number of immunocompromised patients also suggests a poorer immune response in this population. Previous data have already demonstrated that some of these patients had low antibody levels after full vaccination $[13,14]$. In this context, more personalised management of immunosuppressed patients, e.g. measuring antibody levels after vaccination, could prove to be a reasonable option.

Lastly, an increase in comorbidities directly impacts ICU management and the clinical outcomes of a fully vaccinated population. Some studies have already discussed prognosis in patients with previous comorbidities who develop COVID-19 [15, 16]. Indeed, we still observed high ICU mortality rate in fully vaccinated patients, reaching similar levels to previous reports, including those in fully vaccinated patients $[6-8,17,18]$. Worsening of underlying illnesses and/or lower vaccine effectiveness in those patients may provide an explanation for these high rates [8]. Nevertheless, we observed no differences in mortality between both groups, despite higher rates of comorbidities in fully vaccinated patients. Of note, a final decision to not increase supportive measures was made in 16 (19.8\%) fully vaccinated patients.

Our study has some limitations, however. First, we collected data from a small cohort. A larger sample size would be ideal to confer a more robust generalisation of our results. Second, our control group was a small sample of the large, non-vaccinated population. As both study periods partially overlapped, it is also worth considering the role of emerging SARS-CoV-2 variants in these scenarios. Finally, we were not able to know the SARS-CoV-2 viral load and variant, or antibody titres before COVID-19 onset.

To conclude, only $7 \%$ of patients with severe COVID-19 were fully vaccinated. Nonetheless, a clinical scenario of severe COVID-19 disease requiring ICU admission is possible amongst the vaccinated population, especially in those with comorbidities and/or immunosuppression. Therefore, further interventions to improve vaccine response, including an additional dose, might be necessary for this population.

Anna Motos ${ }^{1,2,16}$, Alexandre López-Gavín ${ }^{2,16}$, Jordi Riera $\odot^{3}$, Adrián Ceccato ${ }^{1}$, Laia Fernández-Barat ${ }^{1,2}$, Jesús F. Bermejo-Martin ${ }^{4,5}$, Ricard Ferrer $\oplus^{3}$, David de Gonzalo-Calvo ${ }^{1,6}$, Rosario Menéndez ${ }^{7}$, Raquel Pérez-Arnal ${ }^{8}$, Dario García-Gasulla $\oplus^{8}$, Alejandro Rodriguez ${ }^{9}$, Oscar Peñuelas ${ }^{1,10}$, José Ángel Lorente ${ }^{1,10}$, Raquel Almansa ${ }^{4,5}$, Albert Gabarrus ${ }^{2}$, Judith Marin-Corral ${ }^{11}$, Pilar Ricart ${ }^{12}$, Ferran Roche-Campo ${ }^{13}$, Susana Sancho Chinesta ${ }^{14}$, Lorenzo Socias ${ }^{15}$, Ferran Barbé ${ }^{1,6}$ and Antoni Torres $\circledast^{1,2}$ on behalf of the CIBERESUCICOVID Project (COV20/00110, ISCIII)

${ }^{1}$ Centro de Investigación Biomedica En Red - Enfermedades Respiratorias (CIBERES), Barcelona, Spain. ${ }^{2}$ Institut d'Investigacions August Pi i Sunyer (IDIBAPS), Barcelona, Universitat de Barcelona, Barcelona, Spain. ${ }^{3}$ Intensive Care Dept, Hospital Universitari Vall d'Hebron, Vall d'Hebron Institut de Recerca, Barcelona, Spain. ${ }^{4}$ Hospital Universitario Río Hortega de Valladolid, Valladolid, Spain. ${ }^{5}$ Instituto de Investigación Biomédica de Salamanca (IBSAL), Gerencia Regional de Salud de Castilla y León, Salamanca, Spain. ${ }^{6}$ Translational Research in Respiratory Medicine, Respiratory Dept, Hospital Universitari Aranu de Vilanova and Santa Maria; IRBLleida, Lleida, Spain. ${ }^{7}$ Pulmonary Dept, University and Polytechnic Hospital La Fe, Valencia, Spain. ${ }^{8}$ Barcelona Supercomputing Centre (BSC), Barcelona, Spain. ${ }^{9}$ Critical Care Dept, Hospital Joan XXIII, Tarragona, Spain. ${ }^{10}$ Hospital Universitario de Getafe, Universidad Europea, Madrid, Spain. ${ }^{11}$ Critical Care Dept, Hospital del Mar-IMIM, Barcelona, Spain. ${ }^{12}$ Servei de Medicina Intensiva, Hospital Universitari Germans Trias, Badalona, Spain. ${ }^{13}$ Critical Care Dept, Hospital Verge de la Cinta, Tortosa, Spain. ${ }^{14}$ Servicio de medicina intensive, Hospital Universitario y Politécnico La Fe, Valencia, Spain. ${ }^{15}$ Intensive Care Unit, Hospital Son Llàtzer, Palma de Mallorca, Spain. ${ }^{16}$ A. Motos and A. López-Gavín equally contributed to the manuscript.

Corresponding author: Antoni Torres (atorres@clinic.cat)

Acknowledgements: The authors are indebted to Maria Arguimbau, Raquel Campo, Natalia Jarillo, Javier Muñoz, Elisabeth Sancho and Manuel Sanchez for their extensive support in project management and article preparation. 
CIBERESUCICOVID collaborator group: Berta Adell-Serrano, Alexander Agrifoglio, María Aguilar Cabello, Luciano Aguilera, Victoria Alcaraz-Serrano, Cesar Aldecoa, Cynthia Alegre, Sergio Álvarez, Antonjo Álvarez Ruiz, Rut Andrea, José Ángel, Marta Arrieta, J Ignacio Ayestarán, Joan Ramon Badia, Mariona Badía, Orville Báez Pravia, Ana Balan Mariño, Begoña Balsera, Laura Barbena, Carme Barberà, José Barberán, Enric Barbeta, Tommaso Bardi, Patricia Barral Segade, Marta Barroso, Aaron Blandino Ortiz, José Ángel Berezo García, Judit Bigas, Rafael Blancas, María Luisa Blasco Cortés, María Boado, María Bodi Saera, Neus Bofill, María Teresa Bouza Vieiro, Leticia Bueno, Elena Bustamante-Munguira, Juan Bustamante-Munguira, Jesús Caballero, Lucia Cachafeiro, David Campi Hermoso, Sandra Campos Fernández, losune Cano, Maria Luisa Cantón-Bulnes, Cristina Carbajales, Nieves Carbonell, Pablo Cardina Fernández, Laura Carrión García, Sula Carvalho, Núria Casacuberta-Barberà, Manuel Castellà, Andrea Castellví, Pedro Castro, Mercedes Catalán-González, Ramon Cicuendez Ávila, Catia Cillóniz, Luisa Clar, Cristina Climent, Jordi Codina, Pamela Conde, Sofía Contreras, María Cruz Martin, Maria del Carmen de la Torre, Raul de Pablo Sánchez, Diego De Mendoza, Cecilia del Busto Martínez, Yolanda Díaz, Emili Díaz, María Digna Rivas Vilas, Cristina Dólera Moreno, Irene Dot, Pedro Enríquez Giraudo, Inés Esmorís Arijón, Ángel Estella, Teresa Farre Monjo, Javier Fernández, Carlos Ferrando, Albert Figueras, Eva Forcadell-Ferreres, Lorena Forcelledo Espina, Nieves Franco, Àngels Furro, Cristóbal Galbán, Elena Gallego, Eugenia García, Felipe García, Beatriz García, Emilio García Prieto, Carlos García Redruello, Amaia García Sagastume, José Luis García Garmendia, José Garnacho-Montero, José M. Gómez, Maria Luisa Gascón Castillo, Gemma Gomà, Vanesa Gómez Casal, Silvia Gómez, Carmen Gómez Gonzalez, Jessica González, Federico Gordo, Maria Pilar Gracia, Víctor D. Gumucio- Sanguino, Alba Herraiz, Arturo Huerta, Rubén Herrán-Monge, Mercedes Ibarz, Silvia Iglesias, Maria Teresa Janer, Gabriel Jiménez, Ruth Noemí Jorge García, Mar Juan Díaz, Karsa Kiarostami, Juan I Lazo Álvarez, Miguel León, Ana López Lago, Ana Loza-Vázquez, Desire Macias Guerrero, Nuria Mamolar Herrera, Rafael Mañez Mendiluce, Cecilia L Mantellini, Gregorio Marco Naya, Pilar Marcos, Enrique Marmol Peis, Cinta Marsà-Fadurdo, Paula Martín Vicente, María Martínez, Amalia Martínez de la Gándara, Carmen Eulalia Martínez Fernández, Maria Dolores Martínez Juan, Ignacio Martínez Varela, Juan Fernando Masa Jimenez, Joan Ramon Masclans, Emilio Maseda, Eva María Menor Fernández, Juan Lopez Messa, Mar Miralbés, Josman Monclou, Juan Carlos Montejo-González, Neus Montserrat, María Mora Aznar, Pedro Moral-Parras, Dulce Morales, Sara Guadalupe Moreno Cano, David Mosquera Rodríguez, Guillermo M Albaiceta, Rosana Muñoz-Bermúdez, José María Nicolás, Ramon Nogue Bou, Rafaela Nogueras Salinas, Mariana Andrea Novo, Marta Ocón, Ana Ortega, Sergio Ossa, Pablo Pagliarani, Yhivian Peñasco, Anna Parera Pous, Francisco Parrilla, Leire Pérez Bastida, Purificación Pérez, Gloria Pérez Planelles, Eva Pérez Rubio, David Pestaña Laguna, Àngels Piñol-Tena, Juan Carlos Pozo-Laderas, Javier Prados, Andrés Pujol, Núria Ramon Coll, G loria Renedo Sanchez-Giron, Laura Rodriguez, Felipe Rodríguez de Castro, Silvia Rodríguez, Covadonga Rodríguez Ruiz, Jorge Rubio, Alberto Rubio López, Miriam Ruiz Miralles, Pablo Ryan Murúa, Eva Saborido Paz, Ana Salazar Degracia, Inmaculada Salvador-Adell, Miguel Sanchez, Ana Sánchez, Angel Sánchez-Miralles, Bitor Santacoloma, Maria Teresa Sariñena, Marta Segura Pensado, Lidia Serra, Mireia Serra-Fortuny, Ainhoa Serrano Lázaro, Lluís Servià, Jordi Solé-Violan, Laura Soliva, Carla Speziale, Fernando Suares Sipmann, Luis Tamayo Lomas, Daniel Tognetti, Adrián Tormos, Mateu Torres, Sandra Trefler, José Trenado, Javier Trujillano, Alejandro Úbeda, Luis Urrelo-Cerrón, Estela Val, Luis Valdivia Ruiz, Montse Vallverdú, Maria Van der Hofstadt Martin-Montalvo, Sabela Vara Adrio, Nil Vázquez, Javier Vengoechea, Pablo Vidal Cortes, Clara Vilà-Vilardel, Judit Vilanova, Tatiana Villada Warrington, Hua Yang, Minlan Yang, Ana Zapatero.

Data sharing: The datasets used and/or analysed during the current study are available from the corresponding author per reasonable request.

Author contributions: A. Motos, A. López-Gavín, J. Riera and A. Torres participated in protocol development, study design, study management, statistical analysis and data interpretation, and wrote the first draft of the report. A. Ceccato, L. Fernández-Barat, R. Pérez-Arnal, D. García-Gasulla, O. Peñuelas, J.Á. Lorente, A. Rodriguez, D. de Gonzalo-Calvo, R. Almansa, R. Menéndez, J.F. Bermejo-Martin, R. Ferrer and F. Barbé participated in study design, study management and interpretation, and provided critical review of the first draft of the report. A. Gabarrus performed statistical analysis and provided critical review of the first draft of the report. J. Marin-Corral, P. Ricart, F. Roche-Campo, S. Sancho Chinesta and L. Socias participated in data collection and provided critical review of the first draft of the report. The CIBERESUCICOVID consortium participated in data collection.

Conflict of interest: The authors have disclosed that they do not have any conflicts of interest.

Support statement: Financial support was provided by the Instituto de Salud Carlos III de Madrid (COV20/00110, ISCIII), Fondo Europeo de Desarrollo Regional (FEDER), "Una manera de hacer Europa", and Centro de Investigación Biomedica En Red - Enfermedades Respiratorias (CIBERES). D. de Gonzalo-Calvo has received financial support from the Instituto de Salud Carlos III (Miguel Servet 2020: CP20/00041), co-funded by European Social Fund (ESF)/“Investing in your future". Funding information for this article has been deposited with the Crossref Funder Registry. 
References

1 Ministerio de Sanidad - Gobierno de España. Enfermedad por nuevo coronavirus, COVID-19. www.mscbs.gob. es/profesionales/saludPublica/ccayes/alertasActual/nCov/home.htm Date last updated: 2 October 2021. Date last accessed: 2 October 2021.

2 Bergwerk M, Gonen T, Lustig Y, et al. Covid-19 breakthrough infections in vaccinated health care workers. N Engl J Med 2021; 385: 1474-1484.

3 Tenforde MW, Patel MM, Ginde AA, et al. Effectiveness of SARS-CoV-2 mRNA vaccines for preventing Covid-19 hospitalizations in the United States. Clin Infect Dis 2021; in press [https://doi.org/10.1093/cid/ciab687].

4 Torres A, Arguimbau M, Bermejo-Martin J, et al. CIBERESUCICOVID: a strategic project for a better understanding and clinical management of COVID-19 in critical patients. Arch Bronconeumol 2021; 57: Suppl. 2, 1-2.

5 Baden LR, El Sahly HM, Essink B, et al. Efficacy and safety of the mRNA-1273 SARS-CoV-2 vaccine. N Engl J Med 2021; 384: 403-416.

6 Contou D, Fraisse M, Pajot O, et al. Comparison between first and second wave among critically ill COVID-19 patients admitted to a French ICU: no prognostic improvement during the second wave? Crit Care 2021; 25: 3.

7 Juthani PV, Gupta A, Borges KA, et al. Hospitalisation among vaccine breakthrough COVID-19 infections. Lancet Infect Dis 2021; 21: 1485-1486.

8 Brosh-Nissimov T, Orenbuch-Harroch E, Chowers M, et al. BNT162b2 vaccine breakthrough: clinical characteristics of 152 fully vaccinated hospitalized COVID-19 patients in Israel. Clin Microbiol Infect 2021; 27: 1652-1657.

9 Tenforde MW, Self WH, Adams K, et al. Association between mRNA vaccination and COVID-19 hospitalization and disease severity. JAMA 2021; 326: 2043-2054.

10 Benotmane I, Gautier G, Perrin P, et al. Antibody response after a third dose of the mRNA-1273 SARS-CoV-2 vaccine in kidney transplant recipients with minimal serologic response to 2 doses. JAMA 2021; 326: 1063-1065.

11 Mahase E. Covid-19: third vaccine dose boosts immune response but may not be needed, say researchers. BMJ 2021; 373: n1659.

12 Werbel WA, Boyarsky BJ, Ou MT, et al. Safety and immunogenicity of a third dose of SARS-CoV-2 vaccine in solid organ transplant recipients: a case series. Ann Intern Med 2021; 174: 1330-1332.

13 Boyarsky BJ, Werbel WA, Avery RK, et al. Antibody response to 2-dose SARS-CoV-2 mRNA vaccine series in solid organ transplant recipients. JAMA 2021; 325: 2204-2206.

14 Havlin J, Svorcova M, Dvorackova E, et al. Immunogenicity of BNT162b2 mRNA COVID-19 vaccine and SARS-CoV-2 infection in lung transplant recipients. J Heart Lung Transplant 2021; 40: 754-758.

15 Fang $\mathrm{X}$, Li S, Yu H, et al. Epidemiological, comorbidity factors with severity and prognosis of COVID-19: a systematic review and meta-analysis. Aging (Albany NY) 2020; 12: 12493-12503.

16 Grasselli G, Greco M, Zanella A, et al. Risk factors associated with mortality among patients with COVID-19 in intensive care units in Lombardy, Italy. JAMA Intern Med 2020; 180: 1345-1355.

17 Grasselli G, Zangrillo A, Zanella A, et al. Baseline characteristics and outcomes of 1591 patients infected with SARS-CoV-2 admitted to ICUs of the Lombardy region, Italy. JAMA 2020; 323: 1574-1581.

18 COVID-ICU Group on behalf of the REVA Network and the COVID-ICU Investigators. Clinical characteristics and day-90 outcomes of 4244 critically ill adults with COVID-19: a prospective cohort study. Intensive Care Med 2021; 47: 60-73. 